\title{
A Fast High Precision Skew Angle Estimation of Digitized Documents
}

\author{
Merouane Chettat, Djamel Gaceb, and Soumia Belhadi \\ Laboratory of Computer Science, Modeling, Optimization and Electronic Systems, Faculty of science, \\ University M'hamed Bougara Boumerdes, Algeria
}

\begin{abstract}
In this paper, we treated the problem of automatic skew angle estimation of scanned documents. The skew of document occurs very often, due to incorrect positioning of the documents or a manipulation error during scanning. This has negative consequences on the steps of automatic analysis and recognition of text. It is therefore essential to verify, before proceeding to these steps, the presence of skew on the document to be processed and to correct it. The difficulty of this verification is associated to the presence of graphic zones, sometimes dominant, that have a considerable impact on the accuracy of the text skew angle estimation. We also noted the importance of preprocessing to improve the accuracy and the calculation cost of skew estimation approaches. These two elements have been taken into consideration in our design and development of a new approach of skew angle estimation and correction. Our approach is based on local binarization followed by horizontal smoothing by the Run Length Smoothing Algorithm (RLSA) method, detection of horizontal contours and the Hierarchical Hough Transform (HHT). The algorithms involved in our approach have been chosen to guarantee a skew estimation: accurate, fast and robust, especially to graphic dominance and real time application. The experimental tests show the effectiveness of our approach on a representative database of the Document Image Skew Estimation Contest (DISEC) contest International Conference on Document Analysis and Recognition (ICDAR).
\end{abstract}

Keywords: Skew angle estimation, document images, Hough transform, Binarization, edge detection, RLSA.

\section{Introduction}

Digitization in companies, administrations and libraries represents a real revolution in access to administrative, cultural, scientific and technical heritage. It radically changes the way that we learn, communicate and work. This revolution cannot be achieved on the sole condition that the majority of old or recent documents are digitized in image mode and then converted into editable, searchable and viewable electronic documents on any fixed or mobile device. Optical Character Recognition (OCR) is used to interpret texts in document images and convert them to Unicode format with as few errors as possible from pattern recognition algorithms. The quality of the digitization step of the document (first step in a chain of processing, analysis and automatic recognition of content) is very important because it greatly influences the extraction and recognition of any textual or graphic information in a document image. This is why it is important to take the necessary precautions to scan the document as well as possible. Despite all the precautions taken, during the scanning of a document, a skew of pages (text lines) with respect to the horizontal axis of the image occurs very often. The scanned text may be slightly or heavily skewed (see Figure 1), for various reasons:
- Incorrect manual positioning of the pages in the scanner or handling error

- Paper well placed, but printed or photocopied with skew

- Documents scanned by pocket scanners that must be manually moved on the surface of the page.

- Scanned document using mobile phone cameras or digital cameras (inclined shooting, motion, etc.,)

- Skewed handwritten notes.

- Self-adhesive labels affixed in an skewed manner (sender and destination address, package sending forms, drug sticker, lists of ingredients in food products or chemical components in certain products)

- Skewed text for infographic reasons.

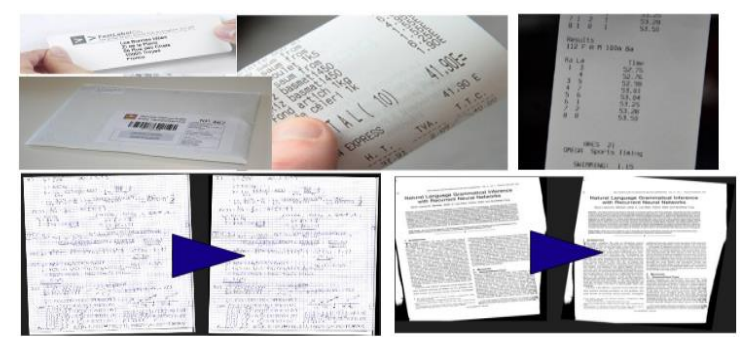

Figure 1. Examples of skewed documents and corrected pages.

This text skew, which affect the quality of scanning documents, can have multiple and serious 
consequences for all stages of automatic content recognition systems: text/graphics separation, text zone location, extraction of the physical, logical or functional layout of documents, hierarchical segmentation of pages to text lines, word or characters, OCR, etc., In practice, a skewed document is often rejected by the automatic reading system when one of its steps fail because the skew of text. Some systems reject the document to be processed when a document skew exceeds five degrees. If the analysis tools do not account for these different skews of text lines, the risk of rejection and read errors OCR increases significantly. To allow optimal localization, extraction and recognition of the text and reduce the rejection rates, it must be corrected any skewed page effectively. The steps skew estimation and correction of document image thus become indispensable before proceeding to the succeeding segmentation and recognition steps. The difficulty lies in the presence of graphic zones (sometimes dominant) that have a considerable impact on the accuracy of the estimation of the skew angle of text. The objective of this work is to develop a technique of skew angle estimation for the automatic document correction that is robust to the presence of graphic elements and degradations and adapted to the real time constraint.

The rest of the paper is organized as follows. The following section presents a brief coverage of the related work. Scetion 3 describes our proposed method of skew estimation of scanned documents. Section 4 presents our experimentation. Finally, we conclude.

\section{Existing Methods for Estimating the Skew Angle of Digitized Documents}

Existing approaches in the literature can be grouped into several main categories according to the basic technique used to estimate the skew angle.

a) Methods based on the projection of profiles of the binary layer of the physical layout. The conventional approach, initially proposed in [14], is based on a sequence of horizontal projection profiles at a range of angles. The angle that offers the maximum variation of the profiles corresponds to the desired skew angle (better alignment of text lines). This method suffers from a very high calculation cost because of its iterative process. For this, several improvements are proposed in $[8,12$, 19, 21]. These improvements remain insufficient on documents with dominance of graphic areas or manuscripts with irregular alignment.

b) Methods based on the Clustering of Connected Components (CCs) using the criterion of nearest neighbours $[4,5,9,11,20]$. These approaches are based on the detection of CCs and the analysis of their alignment. These approaches are sensitive to misalignment of text (manuscript case) and the high presence of graphic areas. Calculation time often exponentially increases with increasing number of CCs.

c) Methods based on linear regression [3, 6, 15]. The linear regression formula is applied on the black pixels of each text line in order to estimate its skew angle, then each skew angle is represented in a histogram of angle and its peak corresponds to the document skew angle. This approach has limitations on the handwritten text, it requires a step of text line segmentation that becomes difficult to the presence of graphic areas.

d) Methods based on the Hough Transform (HT) which determines the straight lines using a black pixels transformation in the polar space $[1,2,7,10$, $16,17,18]$.

After studying these different approaches of skew estimation, the approaches based on the Hough transform were most suited to estimating the skew of entire pages (targeted in our study) containing textual or graphic elements in random directions. On this type of images, the other approaches are either less precise, more expensive in terms of calculation time or dependent on a prior separation of text lines or longitudinal shapes where skew estimation is performed on each. The problem of the conventional Hough approach lies in its consumption of computational times and its sensitivity to the presence of dominant graphic shapes which are directed in irregular orientations. Our goal here is to develop a new TH-based approach that offers a better compromise between computation time and accuracy that can be targeted at a high-speed document processing application.

\section{Proposed Approach}

We present here our skew estimation approach which represents a new alternative to the conventional use of the HT with better speed and accuracy (see the synoptic diagram of Figure 2).

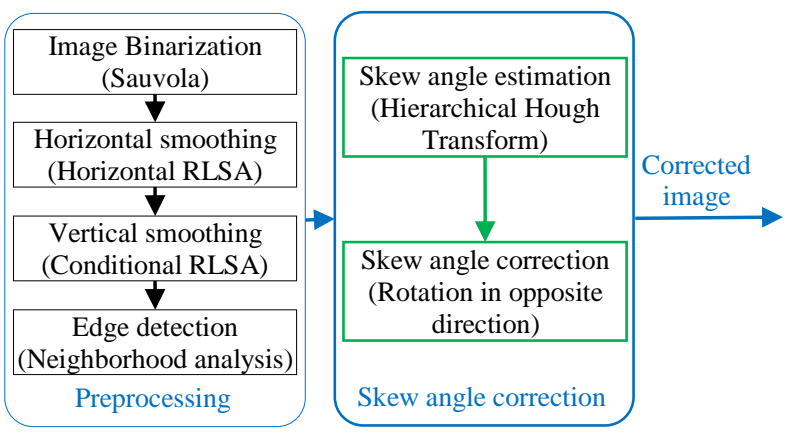

Figure 2. Synoptic of our approach of skew angle estimation.

It is also adapted to images of documents containing some text lines and a lot of graphics. A rotation in the opposite direction of the skew angle is thus used to 
correct the skewed document image. The principal steps of our approach of skew angle estimation are: Pre-processing of the scanned document image and estimation of the skew angle.

\subsection{Preprocessing of the Skewed Image}

This step is very important to address the problem of the complexity of physical layout of image and to facilitate the use of the Hough transform. Without preprocessing, the accuracy of the skew estimation droops on documents with graphic dominance. The preprocessing steps used to prepare the image at the skew angle estimation phase are: Binarization, horizontal smoothing and vertical filtering using Run Length Smoothing Algorithm (RLSA) method and detection of vertical contours.

\subsubsection{Fast Local Binarization}

The binarization (thresholding) is used to convert a grayscale image to a binary black and white image. This transformation should preserve as much relevant information as possible from a document. Its main role is to reduce the large amount of existing information to a simple binary representation that includes only the essential part of this information. It consists in classifying the pixels of a grayscale image according to a threshold in two layers: the foreground, marked in black (integrating all the pixels of the physical structure) and the background in white (integrating all pixels of the background). In the literature there are three main families of methods: global based in general on histogram analysis, local (most used and relevant in practice) based on a local neighborhood analysis and mixed methods (they combine the first two families of methods) [3]. The global methods are fast but their failure is manifested in a considerable way on an image with non-regular illumination. On the other hand, local approaches are more suited to this problem, although their execution time is higher than the first approaches, local methods are most preferred by the digitization industry because of their irrefutable efficiency. It is in this sense that we have chosen to integrate in our approach the smart local and adaptive method presented in [3]. This algorithm is designed to binarize a grayscale image by calculating a local automatic threshold to each pixel according to its local quality and the distribution of luminous intensity in its local neighborhood. The concept of the integral image was used to accelerate significantly the local analysis.

\subsubsection{Horizontal Smoothing and Vertical Filtering Using the RLSA Method}

In our approach, the RLSA is applied line by line (horizontal smoothing) and then column by column (vertical filtering) on the images of documents resulting from the binarization step. The conventional
RLSA algorithm is a very simple and fast algorithm to merge text characters and words into black pixel runs in horizontal direction representing the layout of lines [3]. Our approach uses the RLSA method as follows: Initially, a horizontal RLSA smoothing of the binary image is applied according to a threshold given by the user (it must be large enough to cover the inter-word distance). Subsequently, vertical filtering is applied by removing the fine sequences (runs) of black pixels (width 2 or 3 pixels) which connect two distant blocks which may reduce the accuracy of the skew angle estimation.

a) Horizontal RLSA (Highlight text lines): During this step, we start by fixing a certain threshold $T h$, then the binary image is scanned line by line and pixel by pixel to visit all the image pixels. If the number of (white) background pixels between two foreground (black) pixels is less than or equal to the threshold $T h$, then these white pixels are converted to black pixels. We chose to set the horizontal threshold at the $T h=40$ value at a resolution of $300 \mathrm{dpi}$ (but this depends on the resolution of the image and the size of the text).

b) Filtering of fine vertical lines on the horizontal RLSA result: This operation eliminates fine horizontal lines in the following steps:

- Scan the resulting image from the previous step (horizontal RLSA) column by column.

- At the first black pixel encountered, we begin to calculate the length of the black vertical run.

- When the first white pixel or the end of the column is reached, the calculation of the vertical length of this black run is stopped.

- If this vertical length is lower than a threshold $S_{f v}$ (empirically set to two or three pixels), the pixels of this fine black run are converted to white to remove it. Otherwise, the search for the next black pixel is continued in the same column or in the next column and the step $\mathrm{b}$ is applied.

This filtering allows to avoid the influence of certain fine horizontal lines (in the direction $0^{\circ}$ ) connecting, in a wrong manner, the different lines of text with graphic. These false directions may lower the accuracy of skew angle estimation.

\subsubsection{Detection of Vertical Contours (Neighborhood Analysis)}

This step has an important role to estimate the skew angle, especially on document images with a graphic majority that dominates the text content. The graphic black blocks, resulting from the RLSA smoothing, contain plot lines oriented in random directions. This makes it impossible to estimate the direction of text that is minority. The idea here is to derive the shape of the objects (text or graphic) in vertical contours. This 
step minimizes, considerably, the influence of high number of graphic pixels on the direction estimation of the lines of text. This allows a significant reduction of the calculation times of the next step of the skew angle estimation. To obtain the vertical contours, we have chosen to use a very simple approach based on the analysis of the four horizontal and vertical neighbors ( $3 \times 3$ windo).

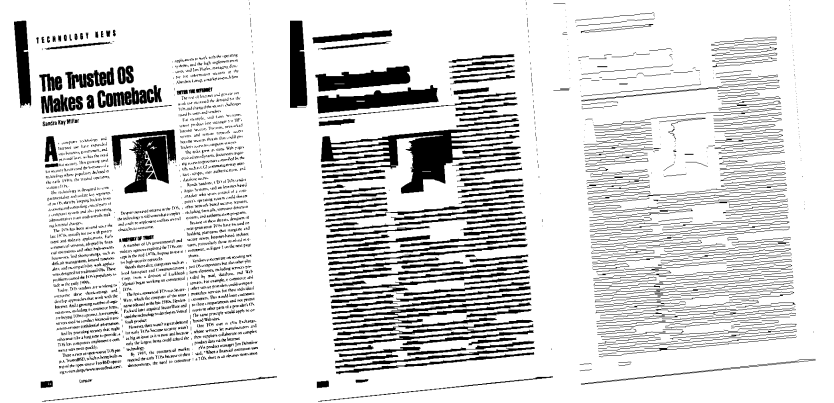

Figure 3. Left: Binary document image inclined at $5^{\circ}$ ( $\left.75 \mathrm{dpi}\right)$, in the center, image resulting from horizontal smoothing by the RLSA method $(S h=15)$, right: the result of the vertical contours detection.

At each black pixel, if its two horizontal neighbors are black and at least one of its two vertical neighbors is white, this pixel is denoted as a point of the vertical contours. The Figure 3 shows an example of detection of the vertical contours on a binary image resulting from the RLSA phase. It can be clearly seen in this figure that this step makes it possible to reduce the impact of the presence of graphic with the text.

\subsection{Skew Angle Estimation Using Hierarchical Hough Transform of Vertical Contours Points}

To estimate the skew angle of text lines, the Hierarchical Hough Transform (HHT) is applied directly to the binary map of the vertical contours. This transform is a well-known technique for estimating skew angle, its estimation accuracy is related to the angular resolution. Increasing this resolution also increases the calculation time. The HT is the transform that allows to pass from the Cartesian coordinate space $(\mathrm{x}, \mathrm{y})$ to the polar coordinate space, using the following formula: $r=x \times \cos \theta+y \times \sin \theta$, any line $D$ in the cartisian space can be characterized by a polar representation with parameters $r$ and $\theta$ such that a point $M(x, y)$ belongs to $D(r, \theta)$ if its coordinates satisfy the relation of the previous equation Figure 4.

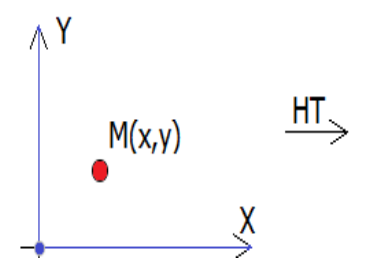

a) Point $\mathrm{M}$ in the original image.

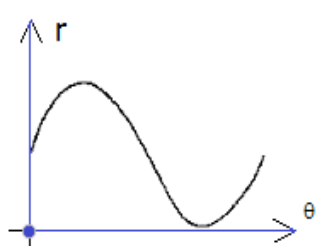

b) Hough transform of $\mathrm{M}$ and its representation in polar space $(\rho, \theta)$.

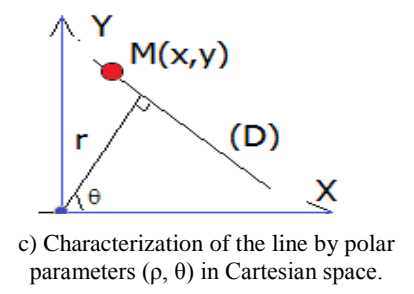

Figure 4. Hough transform of a point M.

Each point $\mathrm{M}$ of the image is characterized by its coordinates $(\mathrm{x}, \mathrm{y})$ which are integers, after the transformation, the points of the transformed space will have the coordinates $\{r, \theta, \operatorname{Acc}(r, \theta)\}$, With $\operatorname{Acc}(r, \theta)$ denoting the number of points of the initial image belonging to the line $D$. The size of the accumulator that we have chosen is of $N_{\theta}$ directions for the angles and of an amplitude of the rays equal to the diagonal of the image ${ }_{A_{\max }}=\frac{1}{2} \sqrt{h^{2}+w^{2}}$, whatever the angle of skew to be detected.
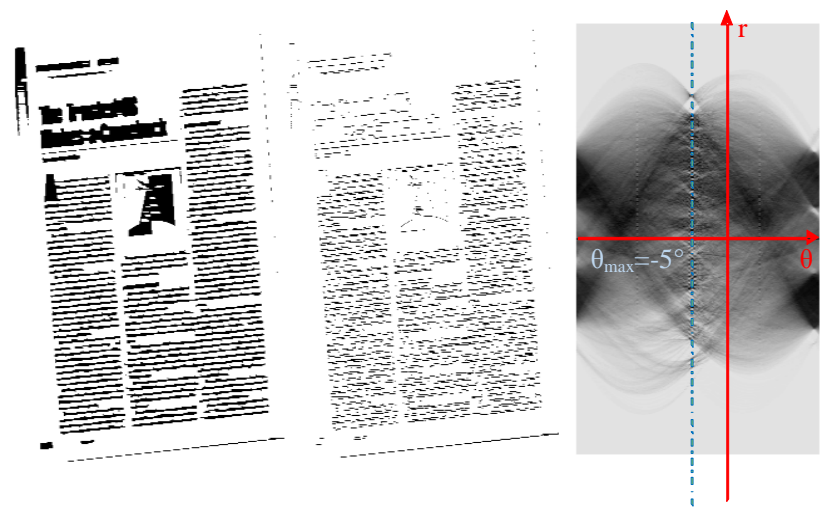

Figure 5. Example of the Hough transform of the vertical contour map. For this document the maximum accumulator has been detected with precision for an angle $\theta=-5^{\circ}$.

The skew angle of text lines corresponds to the maximum value (peak) of the accumulator (see the following Figure 5). To accelerate this step and reduce the size of the search space we use the hierarchy in HT. This approach divides the search interval of the angle $\theta$ into different sub-intervals, the sub-intervals are also divided into angular ranges and so on. The skew angle is searched hierarchically using the HHT. When the angle interval around skew angle is located, a second step divides this interval into several angular ranges which become the new search space on which the HHT is again applied. At each level of hierarchy, the same thing is done and the search of the skew angle is refined until it converges to a desired precision. 
Once the skew angle of a document is detected, the image can be corrected by reverse rotation with the same angle using a three-pass rotation approach [3].

\section{Evaluation and Results}

\subsection{First Evaluation}

The first evaluation of our approach is based on images of the "uva color document data set", database of the University of Amsterdam. These document images are scanned at low resolution (75 dpi). We chose a selection of 30 images with different levels of difficulty (images with colored text and images with textual or graphic domination). An artificial rotation is then applied to each of these images in the following directions: $-25^{\circ},-15^{\circ},-7^{\circ}, 0^{\circ}, 5^{\circ}, 10^{\circ}, 20^{\circ}$ and $37^{\circ}$. We then evaluate the capability of our approach of skew angle estimation to find these directions automatically. This represents a base of 240 images (original images with rotations). See in Figure 6 some examples of images from this dataset:

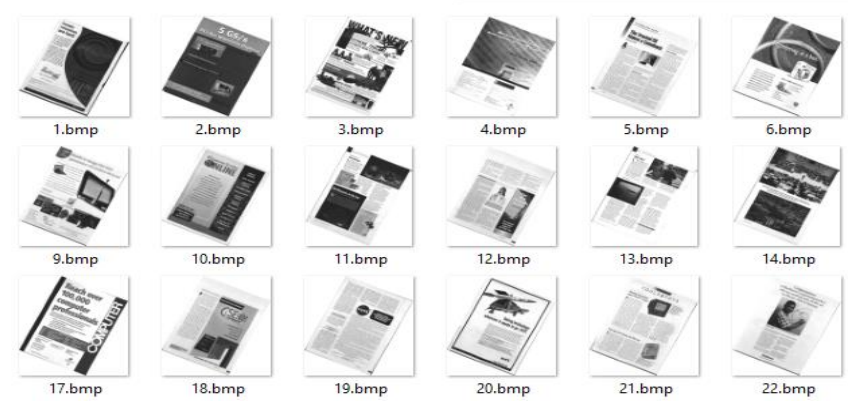

Figure 6. Preview of test images with artificial rotations $-25^{\circ}$.

The Figure 7 shows the percentage of the good detected skew by our approach for each of the 7 orientations $\left(-25^{\circ},-15^{\circ},-7^{\circ}, 0^{\circ}, 5^{\circ}, 10^{\circ}, 20^{\circ}\right.$ and $\left.37^{\circ}\right)$.

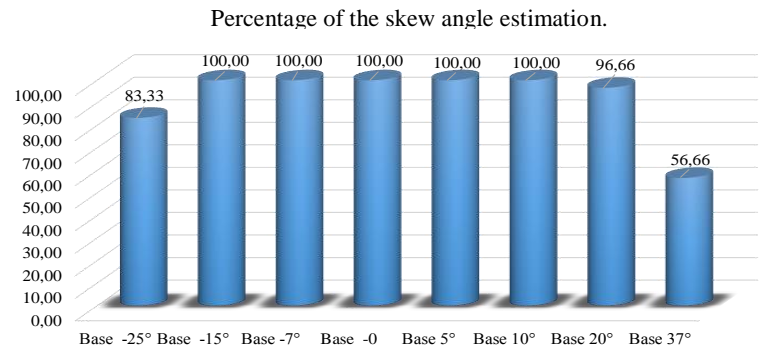

Figure 7. Results of our approach of skew angle estimation on the 240 images of the UVA database, Percentage of the skew angle estimation for each direction.

The results of this evaluation show that the skew estimation in the interval $\left[-15^{\circ},+15^{\circ}\right]$ is at $100 \%$, whatever the difficulty level of the image. This interval actually covers $99 \%$ of the created skew during scanning. For this reason, the competition of the International Conference on Document Analysis and Recognition (ICDAR) Conference was limited to a skew estimation on the interval $\left[-15^{\circ},+15^{\circ}\right]$. We noticed that on the skew angle higher than the limits of this interval (next $45^{\circ}$ ), the ambiguity between the portrait and landscape orientation increases. In this case, the estimated orientation can be orthogonal to the real skew angle, and thus a simple comparison between the height and width of document provides the true orientation of the text lines. This is similar to human perception where the ambiguity between landscape and portrait reaches its maximum at $45^{\circ}$. It should be noted that when the estimated skew angle is orthogonal to the real angle, the corrected document will be read because the OCR applies a reading process adapted to this orthogonal direction. From these remarks we can say that our approach is robust and similar to human perception whatever the complexity of the physical layout of document to be treated.

\subsection{Second Evaluation: Contest DISECof ICDAR Conference}

The second evaluation is based on the image dataset of the skew angle estimation contest (DISEC'13) of the ICDAR International Conference, IEEE [13] (see Figure 8). The database contains 1550 scanned and binarized documents, skewed with arbitrary rotations in the angular range $\left[-15^{\circ},+15^{\circ}\right]$. The following figure shows an overview of the document images of this database.
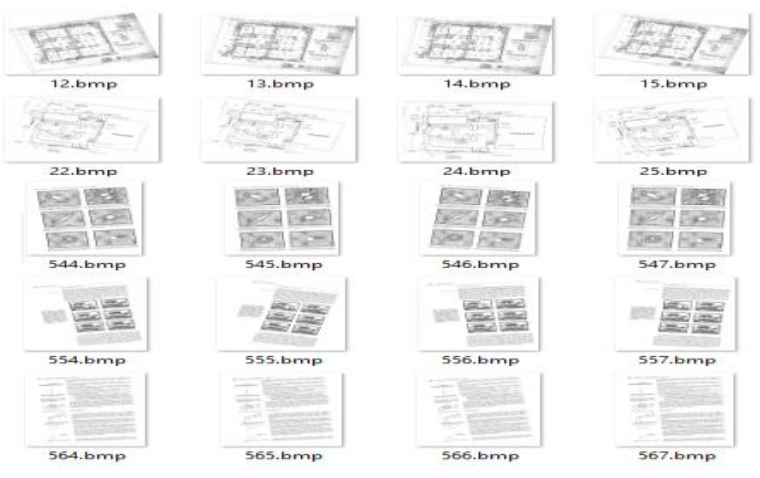

Figure 8. Selection of 1550 documents from the DISEC database.

These documents come from different sources to cover the maximum possible of the reality and contain texts, figures, tables, drawings, graphs, diagrams, synoptic diagrams, architectural plans, electrical circuits. They are collected from newspapers, scientific articles, comics, books, dictionaries, travel guides and museums, administrative and civil records and various other sources. The document images of the datasets are written mainly in English, Chinese and Greek, while there are several documents written in Japanese, Bulgarian, Russian, Danish, Italian, Turkish and Ancient Greek languages. The documents in the database represent: different sizes of document images, any type of mixed content, vertical and horizontal writing, multiple font formats, a multiple number of columns. 
- Performance measures and evaluation: For each document image " $j$ " in the dataset of $N=1550$ documents, the distance $E(j)$ is the distance between the skew angle obtained by our approach and that given by the ground truth. The DISEC' 13 contest protocol required that the estimation of the skew angle be rounded to the second decimal point (Accuracy $=0.1^{\circ}$ ). In order to measure the performance of our method we used the $C E$ criterion (the percentage of correct skew angle estimation) given by the following Equation (1):

$$
C E=\frac{1}{N} \sum_{j=1}^{N} K(j) \text { where } k(j)=\left\{\begin{array}{c}
1 \text { if } E(j) \leq\left(p=0.1^{\circ}\right) \\
0 \text { otherwise }
\end{array}\right.
$$

The threshold of $0.1^{\circ}$ was chosen because the skew angle above this threshold may be visible to a human observer. The following table shows the values of this measures obtained by applying our method on the 1550 images of the DISEC database with a precision $\mathrm{p}=0.1$. The following table shows the ranking of our method in relation to the 12 approaches classified in DISEC using the EC criterion. Details on these approaches are available in [13].

Table 1. Comparison and ranking of our approach compared to the 12 existed methods that have participated in the DISEC' 13 contest using the percentage of correct estimation of skew angle (EC), based on 1550 documents with an angular accuracy $=0.1^{\circ}$.

\begin{tabular}{|c|c|c|}
\hline Methods & CE(\%) & Ranking \\
\hline LRDE-EPITA-a & 77.48 & 1 \\
\hline Our approach & 71.48 & 2 \\
\hline Ajou-SNU & 71.23 & 3 \\
\hline Gamera & 68.90 & 4 \\
\hline LRDE-EPITA-b & 68.32 & 5 \\
\hline HIT-ICG-a & 65.74 & 6 \\
\hline CVL-TUWIEN & 65.42 & 7 \\
\hline HS-Hannover & 58.84 & 8 \\
\hline HP & 58.32 & 9 \\
\hline HIT-ICG-a & 57.29 & 10 \\
\hline CMC_MSU & 50.39 & 11 \\
\hline CST-ECSU & 28.52 & 12 \\
\hline Aria & 19.29 & 13 \\
\hline
\end{tabular}

These results confirm the effectiveness of our approach ranked second according to the criterion CE. The first ranked method (LRDE-EPITA-a) is based on two steps: a preprocessing using a KNN clustering and the skew estimation itself in the frequency domain using the Fourier transform applied to the preprocessing output. The main drawback of this method is the memory and time consumption. Especially, the computation of the k-Nearest Neighbors (KNN) is in $\mathrm{O}(\mathrm{n} 2)$ according to the number of regions in the document. This limitation makes it difficult to apply this method in the context of a realtime application or in a system of high-speed processing of a large document flow (ex. mail or forms sorting).
On the other hand, Our second-ranked approach offers a better speed/precision compromise with a linear complexity $\mathrm{O}(\mathrm{n})$ limited to a reduced number of contour points. It is designed to be easily integrated into a real-time application by taking into account time constraints and result accuracy. The average compute time is $200 \mathrm{~ms}$. The following table shows the percentages of the correct skew angle estimation (using the $C E$ criterion) using our approach according to other precisions $\left(\mathrm{p}=1^{\circ}, \mathrm{p}=0.5^{\circ}, \mathrm{p}=0.3^{\circ}\right)$ on the 1550 images of the Image database.

Table 2. Accuracy of the skew angle estimation on 1550 images of the DISEC database according to other precisions.

\begin{tabular}{|c|c|c|}
\hline $\mathrm{CE}\left(\mathrm{p}=1^{\circ}\right)$ & $\mathrm{CE}\left(\mathrm{p}=0.5^{\circ}\right)$ & $\mathrm{CE}\left(\mathrm{p}=0.3^{\circ}\right)$ \\
\hline $96.32 \%$ & $91.48 \%$ & $79.74 \%$ \\
\hline
\end{tabular}

These results show again the effectiveness of our approach on a wide variety of documents according to different angular precisions $\left(1^{\circ}, 0.5^{\circ}\right.$ and $0.3^{\circ}$ see Figure 9).

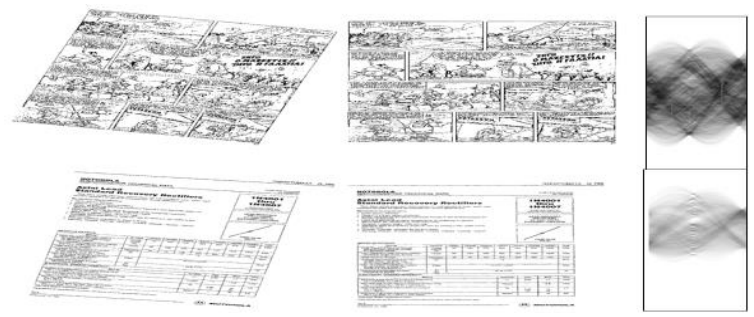

Figure 9. Examples of skew correction on database documents DISEC (left) original skewed image, (center) deskwed image, (right) HT representation in the polar space.

\section{Conclusions}

We presented in this paper our approach of automatic estimation of skew angle on scanned documents. We have demonstrated the contribution of pre-processing for the skew correction especially on documents dominated by graphics and photos. We have shown that our approach offers very interesting precision and reduced computation times. This is thanks to the RLSA approach and the detection of vertical contours which reduces the number of pixels passing through the HHT. This reduction filters a large part of the graphic and increases the robustness of our approach to the presence of graphical objects or random shapes. This robustness has been demonstrated in the tests carried out on the image dataset of the DISEC competition of ICDAR conference. This work represents a real contribution to enrich the real-time OCR applications.

\section{References}

[1] AlKhatatneh A., Pitchay S., and Al Qudah M., "A Review of Skew Detection Techniques for Document," in Proceedings of $7^{\text {th }}$ UKSim-AMSS International Conference on Modelling and Simulation, Cambridge, pp. 316-321, 2015. 
[2] Epshtein B., "Determining Document Skew Using Inter-line Spaces," in Proceedings of International Conference on Document Analysis and Recognition, Beijing, pp. 27-31, 2011.

[3] Gaceb D., Contributions Au Tri Automatique De Documents Et De Courrier D'entreprises, phd, Thesis, INSA de Lyon, 2009.

[4] Hashizume A., Yeh P., and Rosenfeld A., "A Method of Detecting the Orientation of Aligned Components," Pattern Recognition Letters, vol. 4, no. 2, pp. 125-132, 1986.

[5] Jiang X., Bunke H., and Widmer-Kljajo D., "Skew Etection of Document Images By Focused Nearest-Neighbor Clustering," in Proceedings of the $5^{\text {th }}$ International Conference on Document Analysis and Recognition, Bangalore, pp. 629632, 1999.

[6] Kapogiannopoulos G., and Kalouptsidis N.,"A Fast High Precision Algorithm for the Estimation of Skew Angle Using Moments," in Proceedings of Sindh Public Procurement Regulatory Authority, Crete, pp. 275-279, 2002.

[7] Kumar D. and Singh D., "Modified Approach of Hough Transform for Skew Detection and Correction in Documented Images," International Journal of Research in Computer Science, vol. 2, no. 3, pp. 37-40, 2012.

[8] Li S., Shen Q., and Sun J., "Skew Detection Using Wavelet Decomposition and Projection Profile Analysis," Pattern Recognition Letters, vol. 28, pp. 555-562, 2007.

[9] Liolios N., Fakotakis N., and Kokkinakis G., "Improved Document Skew Detection Based on Text Line Connected-Component Clustering," in Proceedings of International Conference on Image Processing, Thessaloniki, pp. 1098-1101, 2001.

[10] Mehta S., Walia E., and Dutta M., "Time and Accuracy Analysis of Skew Detection Methods for Document Images," International Journal of Information Technology and Computer Science, vol.7, pp. 43-54, 2015.

[11] O'Gorman L., "The Document Spectrum for Page Layout Analysis," IEEE Transactions on Pattern Analysis and Machine Intelligenc, vol. 15, no. 11 pp. 1162-1173, 1993.

[12] Papandreou A., and Gatos B., A "Novel Skew Detection Technique Based on Vertical Projections," in Proceedings of International Conference on Document Analysis and Recognition, Beijing, pp. 384-388, 2011.

[13] Papandreou A., Gatos B., Louloudis G., and Stamatopoulos N., "ICDAR2013 Document Image Skew Estimation Contest (DISEC'13)," $12^{\text {th }}$ in Proceedings of Document Analysis and Recognition, Washington, pp. 1444-1448, 2013.
[14] Postl W., "Detection of Linear Oblique Structures and Skew Scan in Digitized Documents," in Proceedings of $8^{\text {th }}$ Pattern Recognition, pp. 687-689, 1986.

[15] Shah L., Patel R., Patel S., and Maniar J., "Skew Detection and Correction for Gujarati Printed and Handwritten Character using Linear Regression," International Journal of Advanced Research in Computer Science and Software Engineering, vol. 4, no. 1, pp. 642-648, 2014.

[16] Shukla B., Kumar G., and Kumar A., "an Approach for Skew Detection Using Hough Transform," International Journal of Computer Applications, vol. 136, no. 9, pp. 20-23, 2016.

[17] Srihari S. and Govindaraju V., "Analysis of Textual Images Using the Hough Transform," Machine Vision and Applications, vol. 2, pp. 141-153, 1989.

[18] Touji S., Ben Amara N., and Amiri1 H., "Generalized Hough Transform for Arabic Printed Optical Character Recognition," The International Arab Journal of Information Technology, vol. 2, no. 4, pp. 326-332, 2005.

[19] Verma R. and Malik L., "Review of Illumination and Skew Correction Techniques for Scanned Documents," in Procedia Computer Science, vol. 45, pp. 322-327, 2015.

[20] Wong K., Casey R., and Wahl F., "Documents Analysis System," IBM Journal of Research and Development, vol. 26, no. 6, pp. 647-656, 1982.

[21] Yildirim B., "Projection Profile Analysis for Skew Angle Estimation of Woven Fabric Images," Journal of the Textile Institute, vol. 105, no. 6, pp. 654-660, 2014.

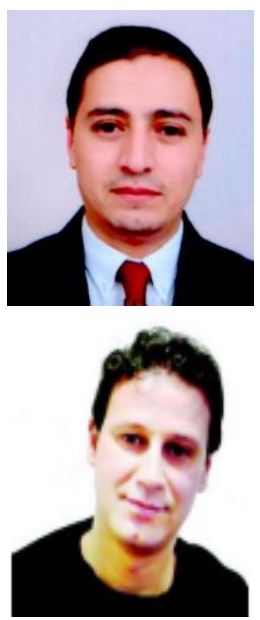

Merouane Chettat is a Ph.D student at UMBB University, Algeria. Currently, he is member of LIMOSE laboratory. His current research interests include image processing, and computer vision.

Djamel Gaceb received his Ph.D. in computer science from INSA of Lyon in 2009. Currently he is working on the topic of business document image recognition, analysis and industrial vision.

Soumia Belhadi is a senior lecturer at the University of Blida. Currently, she is member of LOMOSE Laboratory at UMBB University. She is working on the themes of image processing, and computer vision. 